\title{
Visualizing hypoxia
}

A new method for visualizing hypoxia in prostate cancer tumours, using pixel-wise integration of images that reflect oxygen consumption and supply, has been described in a paper published in Cancer Research. The implementation of this technique - termed consumption and supply-based hypoxia (CSH) imaging - into clinical practice is feasible and could aid treatment decisions in men with this disease.

Overall, 114 men with prostate cancer undergoing radical prostatectomy were included in this study. Patients received pimonidazole, a marker of hypoxia, before surgery and analysis of staining showed that it was significantly associated with clinicopathological features. Comparison of the apparent diffusion coefficient (ADC) and fractional blood volume (fBV) images, which were generated by exploiting intravoxel incoherent motion signal in diffusion-weighted MRI, showed that they correlated with cell density and blood vessel density, respectively, on histological images. Thus, ADC and $\mathrm{fBV}$ can be used as measures of cell density and blood vessel density, respectively, in prostate tumours.

On the basis of this observation, an algorithm was created from the ADC and $\mathrm{ABV}$ images to combine the images of oxygen consumption and supply into a single hypoxia image. A pixel-wise plot was generated for each tumour. An optimal line for discriminating pixels that represented hypoxia was defined using an iterative procedure using data from patients with paired diffusion-weighted image (DWI) and pimonidazole data. The fraction of pixels below the optimized line was used as a measure of the hypoxic fraction predicted by $\mathrm{CSH}$. Hypoxia data was visualized in an image by using the distance of a pixel to the optimized discrimination line to create a hypoxia score for each pixel. This score indicated the possibility that the pixel represented hypoxia and was colour coded. Visually, CSH images showed good agreement with pimonidazole staining.

This CSH imaging technique was validated in an independent cohort of patients with paired DWI and pimonidazole data. The hypoxic fraction was positively associated with lymph node metastasis, tumour stage, Gleason score, and tumour extent. CSH imaging could also be applied to histological cell density and blood vessel images, showing good correlation.

This new method creates images of hypoxia in prostate cancer by using pixel-wise integration of DWIs to visualize oxygen consumption and supply in tumours. These images can also identify aggressive disease and can be applied to histopathology assays. Integrating this imaging modality into the clinic could aid treatment decisions in men with prostate cancer.

ORIGINAL ARTICLE Hompland, T.et al. Combined MR imaging of oxygen consumption and supply reveals tumor hypoxia and aggressiveness in prostate cancer patients. Cancer Res. https://doi.org/10.1158/0008-5472.CAN-17-3806 (2018)

\section{IL-6 mediates high-fat diet- induced tumour growth}

Building upon epidemiological evidence for a link between obesity and prostate cancer progression, new research has identified an IL-6-dependent mechanism of high-fat diet (HFD)-induced tumour growth in a mouse model.

The investigators initially found that immunocompetent mice with prostate-specific Pten-knockout fed a HFD (62.2\% energy from fat) had increased prostate size and weight and $\mathrm{Ki} 67^{+}$tumour cell numbers than control diet (CD; $12.5 \%$ energy from fat)-fed mice. Flow cytometry experiments found that HFD-fed mice harboured a higher tumour-infiltrating myeloid-derived suppressor cell (MDSC) fraction and M2:M1 macrophage polarization ratio than CD-fed mice, inferring a role for local MDSCs and M2 macrophages in HFD-induced inflammation and tumour growth. Indeed, administration of celecoxib (an anti-inflammatory drug) to HFD-fed mice decreased tumour growth and the MDSC and M2 macrophage fractions.

Mechanistic experiments revealed that the HFD increased IL-6 expression in prostate tissues, which was localized in stromal immune cells, primarily $\mathrm{F} 4 / 80^{+} \mathrm{CD} 8^{+}$macrophages, and not tumour cells. Importantly, the HFD increased levels of phosphorylated signal transducer and activator of transcription 3 (pSTAT3) in tumour cells, indicative of paracrine stromal-tumour communication via the IL-6 pathway. Accordingly, treatment with an anti-IL-6 receptor antibody

PROSTATE CANCER

\section{Efficacy of a PSMA-targeted nanoparticle}

A phase II trial has reported the safety and efficacy of BIND-014 - a docetaxel-encapsulating nanoparticle targeted to prostate-specific membrane antigen (PSMA) - in metastatic castration-resistant prostate cancer (mCRPC).

Following a phase I dose-finding study, the phase II trial evaluated BIND-014 $\left(60 \mathrm{mg} / \mathrm{m}^{2}\right.$ every 21 days with prednisone) in 42 chemotherapy-naive patients with $\mathrm{mCRPC}$ who progressed on antiandrogen therapy. The primary end point was radiographic progression-free survival (rPFS), and secondary end points included PSA response and circulating tumour cell (CTC) conversion (defined as a change from baseline of $\geq 5$ to $<5$ CTCs per $7.5 \mathrm{ml}$ of blood). After a median of 6 treatment cycles, 12 of $40(30 \%)$ patients with evaluable data had $\mathrm{a} \geq 50 \%$ PSA decline, and CTC conversion occurred in 13 of 26 (50\%) patients. Of the 19 patients with measurable disease, 9 (47\%) patients had stable disease, 5 (26\%) had partial responses, and $1(0.05 \%)$ had a complete response. Importantly, median rPFS was 9.9 months and 26 of 40 patients ( $65 \%$ ) experienced an rPFS of $\geq 6$ months.

The effect of BIND-014 on PSMA expression on CTCs was also assessed (using an Epic Sciences detection platform) in a subset of 16 patients with detectable CTCs. Eleven (61\%) patients had PSMA ${ }^{+}$ CTCs at baseline, and BIND-014 treatment decreased the median number of PSMA ${ }^{+}$CTCs as early as weeks 3 and 9 , suggesting that the drug was on-target and inducing tumour cell death. "With further validation, one could envision a trial where PSMA expression on CTCs is used as an eligibility criterion to select patients who might benefit from a PSMA-directed agent," explains author Karen Autio. 